\title{
Conhecimento tradicional e usos de copaíba Pela comunidade MaKuXi Darora na Savana de Roraima
}

\section{Rodrigo Leonardo Costa de Oliveira ${ }^{1}$, Luís Felipe Paes de Almeida², Maria Fernanda Berlingieri Durigan ${ }^{3}$, Veridiana Vizoni Scudeller ${ }^{4}$, Reinaldo Imbrozio Barbosa ${ }^{5}$}

\author{
${ }^{1}$ Coordenação de Ciências Biológicas. Universidade Estadual de Roraima. (rodrigo@uerr.edu.br). \\ ${ }^{2}$ Instituto Insikiran. Universidade Federal de Roraima. \\ ${ }^{3}$ Embrapa Roraima. \\ ${ }^{4}$ Instituto de Ciências Biológicas, Universidade Federal do Amazonas. \\ ${ }^{5}$ Instituto Nacional de Pesquisas da Amarônia, Núcleo de Pesquisas de Roraima.
}

Recebido em 03 de junho de 2019. Aceito em 18 de setembro de 2019. Publicado em 26 de setembro de 2019.

Resumo - As espécies de Copaifera L., popularmente conhecidas como copaíba, tem seu óleo-resina utilizado na medicina tradicional indígena. Além disso, sua madeira é apreciada para produção de carvão, lenha ou para construção de casas, devido à sua durabilidade. O objetivo foi investigar o conhecimento tradicional Macuxi e usos da copaíba na comunidade indígena Darora, Terra Indígena São Marcos, Roraima. Foi realizado um inventário, por gênero e idade, sobre o conhecimento da copaíba entre os moradores da comunidade. As entrevistas semiestruturadas foram realizadas com 60 participantes (36 homens e 24 mulheres) entre 18 e 84 anos de idade. Entre estes, 48 participantes (32 homens e 16 mulheres) indicaram o uso de copaíba. A copaíba apresentou 21 usos, principalmente na categoria medicinal (47\% das citações), e para uso de madeira (construção, 33\%, combustível, 7,5\% e tecnologia, 5\%). Embora tenha havido diferenças entre IDV e IEV de homens e mulheres, havia apenas diferenças significativas com homens com mais de 40 anos de idade, indicando que este grupo tem um conhecimento maior sobre o uso de copaíba em relação aos outros participantes, independentemente do gênero e idade $(\mathrm{U}=54,5$ e $\mathrm{p}=0,0075)$.

Palavras-chave: Copaifera pubiflora; Gênero; Idade; Planta Medicinal; Uso Madeireiro; Artesanato; Amazônia.

\section{Traditional KNowledge and usage of copal wood in Darora indigenous community in the RoRaima SavanNa}

Aвstract - The Copaifera L. species, popularly known as copaiba, have its oil-resin used in traditional indigenous medicine. Additionally, its wood is appreciated for charcoal production, as firewood or for house construction, due to its durability. The objective was to investigate the traditional Macuxi knowledge and usage of copaiba in the Darora indigenous community, at São Marcos Indigenous Land, State of Roraima. An inventory was made, by gender and age, about the knowledge on copaiba amongst the community residents. The semi-structured interviews were carried out with 60 participants (36 men and 24 women) between 18 and 84 years of age. Among these, 48 participants ( 32 men and 16 women) indicated the use of copaiba. Copaiba presented 21 uses in Darora community, mainly in the medicinal category (47\% of the quotations), and for timber use (construction, 33\%, fuel, 7.5\% and technology, 5\%). Although there were differences between IDV and IEV among men and women, there were only significant differences with men over 40 years old, indicating that this group has a higher knowledge on copaiba uses in relation to the other participants, regardless of gender and age $(U=54.5$ and $p=0.0075)$.

KeYwords: Copaifera pubiflora; Gender; Age; Medicinal Plant; Timber Usage; Handcraft; Amazon region. 


\section{Uso y conocimiento de la copaíba por la comunidad MaKuXi Darora en la Savana de Roraima}

RESUMEN - Las especies de Copaifera L., popularmente conocidas como copaíba, tienen su oleorresina empleado en la medicina tradicional indígena. Su madera es también apreciada para la producción de carbón y leña, así como en la construcción civil por su característica de durabilidad. El objetivo fue investigar el conocimiento y usos tradicionales de la copaíba en la Comunidad Indígena Darora de la etnia Makuxi, en la Tierra Indígena São Marcos, en el estado de Roraima. Se realizó un inventario, por género y edad, del conocimiento sobre esta planta entre los habitantes de la comunidad. Las entrevistas semiestructuradas fueron realizadas con 60 participantes (36 hombres y 24 mujeres), entre 18 y 84 años. De estos, 48 participantes (32 hombres y 16 mujeres), indicaron usos asociados a la copaíba. La copaíba presentó 21 usos, principalmente en la categoría medicinal (el $47 \%$ de las citas) y en cuanto al uso maderero (construcción el 33\%, combustible el 7,5\% y tecnología el 5\%). Aunque existen diferencias entre los valores de IDV y IEV entre hombres y mujeres, sólo hubo diferencias significativas con respecto a los hombres con más de 40 años, indicando que este grupo tiene mayor conocimiento sobre el uso de la copaíba con respecto a los demás participantes, independiente del género y edad $(\mathrm{U}=54,5$ y p $=0,0075)$.

Palabras Clave: Copaifera Pubiflora; Género; Edad; Planta Medicinal; Uso Maderero; Artesanía; Amazonia.

\section{INTRODUÇÃO}

As espécies do gênero Copaifera (Fabaceae) são muito conhecidas pelo potencial medicinal de seu óleo-resina, que é usado desde o período colonial do Brasil. O óleo-resina de copaíba apresenta amplo uso na medicina popular, indígena e na indústria farmacêutica (Rigamonte Azevedo et al. 2004). Copaifera ocorre na África (4 spp.), e existe uma provável espécie na Ásia, e principalmente nas Américas Central (4 spp.) e do Sul (cerca de 37 spp.), as quais, 9 ocorrem na região Amazônica (Martins-da-Silva et al. 2008). No Brasil, ocorre em todas as regiões (Costa 2007; 2019). Existe grande interesse na madeira de algumas espécies do gênero, devido a sua superfície lisa, lustrosa, durável, de alta resistência ao ataque de xilófagos e baixa permeabilidade, características desejáveis para o uso na fabricação de peças torneadas e para a marcenaria em geral (Carvalho 1994). A madeira é bastante utilizada para a fabricação de carvão, e pelas indústrias de construção civil e naval (Veiga Junior e Pinto 2002).

A utilização de espécies do gênero Copaifera é tão intensificada que baseado numa classificação que seguiu critérios de pressão antrópica, frequência de mercado e demanda, estas foram incluídas entre as espécies medicinais e aromáticas prioritárias para a conservação do Bioma Amazônia, durante a $1^{\text {a }}$ Reunião Técnica Sobre Recursos Genéticos de Plantas Medicinais e Aromáticas, a qual reuniu profissionais e instituições de todo o Brasil a partir de uma importante iniciativa da EMBRAPA e do IBAMA. (Vieira et al. 2002).

Em Roraima, a principal espécie encontrada é Copaifera pubiflora Benth., encontrada em ambientes florestais da savana, como Mata Ciliar e Ilha de Mata, tendo sua ocorrência também na Venezuela e Guiana. Apesar das espécies de Copaifera apresentarem múltiplos usos, a ausência de estudos etnobotânicos quantitativos na região é iminente e impossibilita o desenvolvimento de estratégias efetivas quanto à conservação local das espécies. Associado a isto, dois fatores existentes nas savanas de Roraima ameaçam a conservação das espécies: (i) a modificação intensa que Roraima savana sofreu devido a monoculturas e florestação de espécies exóticas (Aguiar Jr et al. 2014) e (ii) o deslocamento da maioria dos jovens em busca de emprego e melhores instalações nos centros urbanos próximos (Heck et al., 2005), que podem alterar diretamente na estrutura da comunidade indígena referentes à transmissão do conhecimento entre os moradores.

O nordeste de Roraima compreende a maior área de savana natural da Amazônia brasileira localmente conhecida como "lavrado", "campos do rio Branco" ou "campos de Roraima", abrangendo 43.000 km² de área (Barbosa e Campos 2011). A savana de Roraima faz parte do complexo de vegetação Rio Branco-Rupununi, que se estende da Venezuela para a República da Guiana (Barbosa e Fearnside 2005), formando um grande mosaico de 
ecossistemas não-florestais (vegetação aberta com baixa densidade de árvores) e florestais, como matas de galeria e buritizais), com dominância de áreas de vegetação aberta (Barbosa et al. 2007).

Nesta enorme área de vegetação aberta, várias terras indígenas são encontradas, a qual as Terra Indígena Raposa/ Serra do Sol e a Terra Indígena São Marcos destacam-se por suas proporções. As áreas florestais da savana apresentam espécies fundamentais para uso das comunidades indígenas, como importantes fontes alimentícia e medicinal, bem como de uso madeireiro para fins de construção, combustível, tecnologias e outros. Na comunidade Darora também foi realizado o estudo de uso e conhecimento dos mirixis (Byrsonima crasssifolia e B. coccolobifolia) na perspectiva de gênero e idade dos participantes. Em relação à estas espécies, não houve diferenças significantes entre as classes etárias e de gênero (Oliveira et al. 2017a).

Sendo assim, este trabalho objetivou analisar o uso e o conhecimento de C. pubiflora entre os moradores da Comunidade Makuxi Darora, correlacionando gênero e classes etárias dos participantes.

\section{Material e métodos}

A Terra Indígena de São Marcos (TISM) compreende cerca de 654.110 ha e 42 comunidades indígenas das etnias Makuxi, Taurepang e Wapixana. O presente estudo envolveu um grupo da etnia Makuxi da comunidade Darora, que vive em uma região conhecida como Baixo São Marcos (3 $10^{\prime} 42$ “N; 60 23’34” W). A área de estudo fica a $90 \mathrm{~km}$ da capital do estado, Boa Vista. Segundo a classificação de Köppen, o clima local é do tipo Aw, característico da savana tropical. A temperatura média anual de $27,8^{\circ} \mathrm{C}$ e uma precipitação média anual de $\sim 1650 \mathrm{~mm}$. De acordo com Barbosa (1997), o período mais seco concentra-se entre os meses de dezembro e março ( $\pm 9 \%$ de precipitação anual), e o período mais úmido entre maio e agosto ( $\pm 70 \%$ de precipitação anual). Atividades de agricultura e pecuária animal caracterizam-se de grande aptidão na Comunidade. Ambas são tidas por eles como necessária para alimentação e sustento. Atividades extrativistas também são observadas e estão sempre relacionadas à construção de casas, cercas e coleta de frutas.

De acordo com os dados do posto de saúde da Comunidade, 40 famílias e 184 pessoas vivem em Darora, das quais 50 são homens e 43 mulheres com mais de 18 anos; e cinco são homens e seis são mulheres com mais de 60 anos de idade. Dentre os residentes citados pelos participantes, nove homens e 12 mulheres não foram encontrados, já que muitos deles se mudam da comunidade para a cidade de Boa Vista, buscando emprego e educação contínua. Para esta pesquisa, apenas uma mulher se recusou a participar. Todos os residentes falam fluentemente o português. A língua Makuxi, do tronco lingüístico Karib, é ensinada na pré-escola, mas raramente é falada pelos moradores, com apenas dois professores e um falante idoso.

\section{Levantamento etnobotânico}

Para a coleta de dados etnobotânicos foram realizadas entrevistas semiestruturadas com 60 informantes (36 homens e 24 mulheres) entre 18 e 84 anos de idade. Foram visitadas todas as residências na comunidade, onde os moradores presentes eram convidados a participar da pesquisa. Neste momento, foi explanado sobre a finalidade da pesquisa e apresentado o Termo de Consentimento Livre e Esclarecido (TCLE), que foi assinado por todos. Na entrevista, os informantes foram direcionados a indicar o conhecimento e usos atribuídos às espécies.

As entrevistas foram realizadas entre novembro de 2014 e novembro de 2015. Num primeiro momento, os entrevistados indicaram os locais de coleta das espécies estudadas em áreas não florestais de savana, e então a técnica de lista livre foi usada para as espécies que eles conheciam na sua nesses ambientes (Albuquerque et al. 2014). Copaíba foi uma das espécies mais citadas pelos moradores. Em outro momento, foram realizadas entrevistas semi-estruturadas com perguntas relacionadas à coleta, formas de uso, e partes das plantas utilizadas. 
As respostas foram agrupadas nas categorias de uso adaptadas de estudos anteriores (Lins Neto et al. 2008; Lucena et al. 2012).

Na comunidade Darora foi encontrada a espécie Copaifera pubiflora, única espécie do gênero Copaifera nesta região do estado de Roraima, localmente chamada de copaíba, a qual uma amostra foi coletada e após processo de herborização foi tombada no Herbário da Universidade Federal de Roraima (UFRR), sob o número de tombo 8454. A identificação ocorreu com o auxílio de guias de flora da região e auxílio de especialista (Flores e Rodrigues 2010).

Quanto ao conhecimento e uso das espécies entre homens e mulheres de diferentes idades foi analisado para reconhecer que fatores podem afetar a transmissão do conhecimento. Os dados obtidos nas entrevistas foram correlacionados entre os gêneros e em dois grupos de idade dos informantes $(<40$ anos e $\geq 40$ anos de idade), com o intuito de analisar a transmissão do conhecimento e uso das espécies por meio de duas medidas quantitativas: $\mathrm{O}$ valor de diversidade do informante (IDV), que representa o número de uso-citações dados por um informante $(\mathrm{Ux})$ dividido pelo total do número total de usos, foi utilizado para avaliar quantos informantes usaram esta espécie e como o conhecimento estava distribuído entre os informantes. O valor de equitabilidade (IEV) do informante equivaleu cada valor de diversidade do informante (IDV) dividido pelo maior valor de diversidade do informante encontrado (IDV max). Esta variável mediu o grau de homogeneidade entre o conhecimento dos informantes (Byg e Balslev 2001). Os informantes foram agrupados conforme o gênero e categorias de idade. A categoria $1,<40$ anos e categoria $2, \geq 40$ anos. Para verificar diferenças entre os valores e categorias foi utilizado o teste Mann-Whitney.

\section{Aspectos legais da pesquisa}

Este estudo é parte do projeto de pesquisa intitulado "Uso e conservação dos recursos vegetais de comunidades indígenas no norte de Roraima”, e foi submetido e aprovado pelo Comitê de Ética em Pesquisa com Seres Humanos do Instituto Nacional de Pesquisas da Amazônia (CEP-INPA/CONEP) sob o número 814.370; e autorizado pela Fundação Nacional do Índio (FUNAI): Processo 08620.002869/2014-15 e pelo Instituto do Patrimônio Histórico e Artístico Nacional (IPHAN): Processo 01450.001678/2014-88.

\section{Resultados E Discussão}

Copaifera pubiflora é uma espécie amplamente conhecida pelos indígenas da Comunidade Darora. Dos 60 moradores entrevistados, 48 (32 homens e 16 mulheres) indicaram 21 usos associados à espécie. A categoria < 40 anos incluiu seis mulheres e 13 homens; a categoria 2, $\geq 40$ anos, 10 mulheres e 19 homens. Embora existam diferenças entre os valores de IDV e IEV entre homens e mulheres, houve apenas diferenças significativas em relação aos homens $\geq 40$ anos, indicando que este grupo detém maior conhecimento sobre o uso da copaíba na Comunidade Darora em relação aos demais participantes, independente do gênero e idade $(\mathrm{U}=54,5$ e p = 0,0075) (Tabela 1). Também foram realizadas análises entre categorias etárias com intervalos de 10 anos, as quais não apresentaram diferenças significativas entre as mulheres e entre os homens. Em estudos realizados em ambientes áridos e semi-áridos, o conhecimento sobre espécies de uso alimentício e medicinal foi uniforme entre os gêneros e classes etárias (Lins Neto et al. 2010; Feitosa et al. 2014). Por outro lado, Voeks (2007), relaciona que o conhecimento entre homens e mulheres varia de acordo com as atividades diárias realizadas para cada gênero, as mulheres como realizam atividades domésticas seriam maiores conhecedoras de espécies medicinais e alimentícias; já os homens, como trabalham na área externa, seriam maiores conhecedores do uso madeireiro das espécies locais. Fato este, também registrado por Monteiro et al. (2005) e Silva et al. (2011), tais divisões de trabalho entre homens e mulheres também foram observadas na comunidade Darora. 
Tabela 1. Medidas de conhecimento da copaíba (Copaifera pubiflora Benth.) na Comunidade Darora (Boa Vista, Roraima, Brasil). IDV - Valor de diversidade do informante, IEV - Valor de equitabilidade do informante.

\begin{tabular}{lc}
\hline Variáveis & Valores \\
\hline Total de informantes & 48 \\
Número de citações de uso & 189 \\
Tipos de uso & 21 \\
\hline Medidas & Média e Desvio-padrão \\
\hline IDV Total & (X \pm SD) \\
IDV Total Mulheres & $1.752 \pm 0.125$ \\
IDV Mulheres $(<40$ anos $)$ & $2.560 \pm 4.534$ \\
IDV Mulheres $(\geq 40$ anos $)$ & $3.155 \pm 5.335$ \\
IDV Total Homens & $2.203 \pm 4.252$ \\
IDV Homens $(<40$ anos $)$ & $1.348 \pm 1.150$ \\
IDV Homens $(\geq 40$ anos $)$ & $1.895 \pm 1.173^{\mathrm{a}}$ \\
IEV Total & $0.974 \pm 0.999^{\mathrm{b}}$ \\
IEV Total Mulheres & $2.787 \pm 0.199$ \\
IEV Mulheres $(<40$ anos $)$ & $0.182 \pm 0.323$ \\
IEV Mulheres $(\geq 40$ anos $)$ & $0.225 \pm 0.38$ \\
IEV Total Homens & $0.157 \pm 0.303$ \\
IEV Homens $(<40$ anos $)$ & $0.096 \pm 0.082$ \\
IEV Homens $(\geq 40$ anos $)$ & $0.135 \pm 0.083^{\mathrm{a}}$ \\
\hline
\end{tabular}

Dos 21 diferentes usos para copaíba nas categorias medicinal, alimentação construção, combustível, tecnologia e artesanato (Tabela 2), apenas uma citação foi relacionada para fins artesanais, que consiste no uso das sementes para confecção de colares. Para o uso alimentício (7,5\% das citações), foram indicados o consumo in natura do óleo. Na perspectiva alimentícia, o óleo da copaíba é difundido como aditivo de alimentos com aprovação pelo FDA (Food and Drugs Administration) (Cascon 2004). Nesta mesma proporção de citações (7,5\%), foi indicado o uso como fonte de combustível, sob a forma de lenha e carvão. $\mathrm{Na}$ Comunidade Darora, a copaíba é usada como fonte de energia nos fornos para produção da farinha, juntamente com as espécies de mirixis, Byrsonima crassifolia e B. coccolobifolia (Oliveira et al. 2017b), que têm seu uso como lenha bastante difundido na utilização doméstica. Para Monteiro et al. (2005), as áreas áridas e semi-áridas são caracterizadas por plantas usadas como combustível, uma vez que o gás engarrafado de uso doméstico é caro ou difícil de obter regularmente para cozinhar alimentos. O uso da copaíba para fabricação de carvão também foi registrado no levantamento bibliográfico realizado por Veiga Junior e Pinto (2002).

O uso da madeira para fins tecnológicos, confecção de tábuas e canoas, corresponderam a 4\% das citações, e para fins de construção (33\%), das quais, $63 \%$ foram citados para o uso de estaca-de-casa e cerca, além do uso chamado pelos participantes de "madeira do ar", onde são indicados usos para a construção do telhado (caibro, linha, ripa e outros), que na maioria dos casos é finalizado com a palha do buriti (Mauritia flexuosa L. f.), encontrado nos buritizais da região. O uso madeireiro total compreendeu $52 \%$ das citações de uso. Este uso das espécies de Copaifera é considerado bastante comum e encontrado na literatura por apresentar propriedades em marcenaria, carpintaria, construção civil e naval (Carvalho 1994; Cascon 2004).

O uso medicinal compreendeu $47 \%$ das citações, destes, 39\% foram relacionadas ao uso para inflamação geral, 26\% para ferimentos e $20 \%$ para diarreia. A casca do caule e o óleo foram as partes da planta mais indicadas com $45 \%$ das citações medicinais. Infecção urinária, inflamação uterina, gripe/pneumonia e atividade vermífuga também foram citados. Quanto ao potencial de uso do óleo de Copaifera, atividades anti-inflamatória e cicatrizante, 
antimicrobiana (Diefenbach et al. 2017; Lucca et al. 2018; Martins-da-Silva et al. 2008). Estudos relatam o uso antihemorroidal e purgativo do chá das cascas e sementes de Copaifera na Venezuela e na Colômbia, e o tratamento de moléstias pulmonares e asma na Amazônia Brasileira (Carvalho, 1994; Veiga Junior e Pinto 2002). Embora exista o grande potencial do uso do óleo pelos moradores, em Darora parece não existir uma atenção especial de conservação da espécie. O uso madeireiro da copaíba como lenha para a torra da farinha apresenta-se como uma atividade extremamente danosa, onde todo o indivíduo é removido para tal e corriqueiramente observada.

Tabela 2. Categorias de uso e partes usadas de copaíba (Copaifera pubiflora Benth.) na Comunidade Darora (Boa Vista, Roraima, Brasil). Número de participantes: 48 (32 homens e 16 mulheres). Categorias de idade < 40: seis mulheres e 13 homens; $\geq 40$ : 10 mulheres e 19 homens).

\begin{tabular}{|c|c|c|c|c|c|c|c|}
\hline \multirow{3}{*}{$\begin{array}{l}\text { Categorias } \\
\text { de uso }\end{array}$} & \multirow{3}{*}{ Usos } & \multirow{3}{*}{ Parte da planta } & \multirow{3}{*}{$\begin{array}{l}\text { Número } \\
\text { de } \\
\text { citações }\end{array}$} & \multicolumn{4}{|c|}{$\begin{array}{l}\text { Número de citações/ categorias } \\
\text { de idade e gênero }\end{array}$} \\
\hline & & & & \multicolumn{2}{|c|}{ Mulheres } & \multicolumn{2}{|c|}{ Homens } \\
\hline & & & & $<40$ & $\geq 40$ & $<40$ & $\geq 40$ \\
\hline \multirow{15}{*}{ Medicinal } & \multirow{4}{*}{ Inflamação geral } & Casca & 18 & \multirow{15}{*}{11} & \multirow{15}{*}{20} & \multirow{15}{*}{17} & \multirow{15}{*}{41} \\
\hline & & Entrecasca & 3 & & & & \\
\hline & & Folha & 1 & & & & \\
\hline & & Óleo & 13 & & & & \\
\hline & \multirow{3}{*}{ Ferimentos } & Casca & 8 & & & & \\
\hline & & Entrecasca & 2 & & & & \\
\hline & & Óleo & 13 & & & & \\
\hline & \multirow{3}{*}{ Diarreia } & Casca & 10 & & & & \\
\hline & & Fruto & 3 & & & & \\
\hline & & Óleo & 6 & & & & \\
\hline & Infecção urinária & Casca & 2 & & & & \\
\hline & Inflamação uterina & Óleo & 3 & & & & \\
\hline & \multirow{2}{*}{ Gripe/Pneumonia } & Casca & 1 & & & & \\
\hline & & Óleo & 5 & & & & \\
\hline & Vermífugo & Casca & 1 & & & & \\
\hline \multirow{7}{*}{ Construção } & Estaca-de-casa & Caule & 36 & \multirow{7}{*}{6} & \multirow{7}{*}{14} & \multirow{7}{*}{16} & \multirow{7}{*}{26} \\
\hline & Estaca-de-cerca & Caule & 3 & & & & \\
\hline & Caibro & Caule & 9 & & & & \\
\hline & Linha & Caule & 7 & & & & \\
\hline & Ripa/ Ripão & Caule & 3 & & & & \\
\hline & Travessa & Caule & 1 & & & & \\
\hline & Perna-manca & Caule & 3 & & & & \\
\hline \multirow{2}{*}{ Alimentícia } & Consumo In natura & Fruto & 12 & \multirow{2}{*}{3} & \multirow{2}{*}{5} & \multirow{2}{*}{3} & \multirow{2}{*}{3} \\
\hline & Suco & Fruto & 2 & & & & \\
\hline \multirow{2}{*}{ Combustível } & Lenha & Caule & 9 & \multirow{2}{*}{2} & \multirow{2}{*}{3} & 2 & 7 \\
\hline & Carvão & Caule & 5 & & & & \\
\hline Tecnolooin & Tábua & Caule & 8 & 0 & 4 & 0 & 5 \\
\hline 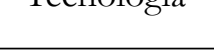 & Canoa & Caule & 1 & 0 & $T$ & 0 & 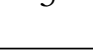 \\
\hline Artesanato & Colar & Semente & 1 & 0 & 1 & 0 & 0 \\
\hline
\end{tabular}




\section{ConClusões}

A copaíba (Copaifera pubiflora) é usada para diferentes finalidades nas categorias medicinal (47\% das citações), alimentícia (7,5\%), e principalmente às relacionadas ao uso madeireiro (construção, 33\%, combustível, 7,5\% e tecnologia, 5\%) na comunidade Darora. Os homens mais velhos ( $\geq 40$ anos) detêm maior conhecimento sobre o uso da espécie em relação aos demais participantes independente de gênero e classes etárias.

\section{REFERÊNCIAS}

Aguiar JR, A, Barbosa, RI, Barbosa, JBF, Mourão JR, M. 2014. Invasion of Acacia mangium in Amazonian savannas following planting for forestry. Plant Ecology and Diversity, 7: 359-369.

Albuquerque, UP, Lucena, RFP, Lins-Neto, EMF. 2014. Selection of research participants. In: Methods and techniques in Ethnobiology and Ethnoecology. In: Albuquerque, UP.; Cunha, LVFC.; Lucena, RFP.; Alves, RRN. (Eds), Springer Protocols Handbooks, New York, p. 1-13.

Barbosa, RI. 1997. Distribuição das chuvas em Roraima. In: Barbosa, R.I., Ferreira, E.J., Castellon, E.G. (eds.) Homem, Ambiente e Ecologia no Estado de Roraima. Instituto Nacional de Pesquisas da Amazônia. Manaus, Amazonas, p. 325 -335 .

Barbosa, RI, Campos, C. 2011. Detection and geographical distribution of clearing areas in the savannas ('lavrado') of Roraima using Google Earth web tool. Journal of Geography and Regional Planning, 4: 122-136.

Barbosa, RI, Campos, C, Pinto, F, Fearnside, PM 2007. The "Lavrados" of Roraima: Biodiversity and Conservation of Brazil's Amazonian Savannas. Functional Ecosystems and Communities, 1(1): 29-41.

Barbosa, RI, Fearnside, PM. 2005. Fire frequency and area burned in the Roraima savannas of Brazilian Amazonia. Forest ecology and management, 204: 371-384.

Byg, A, Balslev, H. 2001. Diversity and use of palms in Zahamena, eastern Madagascar. Biodiversity and Conservation, 10: 951-970.

Carvalho, PER. 1994. Espécies florestais brasileiras: recomendações silviculturais, potencialidades e uso de madeira. Brasilia: EMBRAPA/CNPF, 640p.

Cascon, V. 2004. Copaíba - Copaifera spp. In: CARVALHO, J.C.T. Fitoterápicos antiinflamatórios: aspectos químicos, farmacológicos e aplicações terapêuticas. Ribeirão Preto: Tecmedd, 480p.

Costa, JAS. 2007. Estudos taxonômicos, biossistemáticos e filogenéticos em Copaifera L. (Leguminosae-Detarieae) com ênfase nas espécies do Brasil extra-amazônico. Tese de doutorado (Botânica). Universidade Estadual de Feira de Santana. 249p. 2007

Costa, JAS. 2019. Copaifera in Flora do Brasil 2020 em construção. Jardim Botânico do Rio de Janeiro.Disponível em: <http://reflora.jbrj.gov.br/reflora/floradobrasil/FB22895>. Acesso em: 26 Ago. 2019

Diefenbach, AL, Muniz, FWMG, Oballe, HJR, Rösing, CK. 2017. Antimicrobial activity of copaiba oil (Copaifera spp.) on oral pathogens: systematic review. Phytotherapy Research, doi: 10.1002/ptr.5992 
Feitosa, IS, Albuquerque, UP, Monteiro, JM. 2014. Knowledge and extrativism of Stryphnodendron rotundifolium Mart. in a local community of the Brazilian Savanna, Northeastern Brazil. Journal of Ethnobiology and Ethnomedicine, 10: 64.

Flores AS, Rodrigues, RS. 2010. Diversidade de Leguminosae em uma área de savana do estado de Roraima, Brasil. Acta

Botanica Brasilica, 24: 175-183.

Francisco, SG. 2005. Uso do óleo de copaíba (Copaifera officinalis) em inflamação ginecológica. Femina, 33(2): 89-93.

Heck, E, Loebens, F, Carvalho, PD. 2005. Amazônia indígena: conquistas e desafios. Estudos Avançados, 19: 237-255.

Lins Neto, EMF, Peroni, N, Albuquerque, UP. 2010. Traditional knowledge and management of umbu (Spondias tuberosa, Anacardiaceae): an endemic species from the semi-arid region of Northeastern Brazil. Economic Botany, 64: 11-21.

Lins Neto, EMF, Ramos, MA, Oliveira, RLC, Albuquerque, UP. 2008. The Knowledge and harvesting of Myracrondruon urundewva Allemão by Two Rural Communities in NE Brazil. Functional Ecosystems and Communities, 2: 66-71.

Lucca, L, Matos, S, Kreutz, T, Teixeira, H, Veiga-Júnior, VF, Araújo, B, Limberger, R, Koester, L. 2018. Anti-inflammatory Effect from a Hydrogel Containing Nanoemulsified Copaiba oil (Copaifera multijuga Hayne).AAPS Pharm Sci Tech, 19: 522530.

Lucena, RFP, Medeiros, PM, Araújo, EL, Alves, AGC, Albuquerque, UP. 2012. The ecological apparency hypothesis and the importance of useful plants in rural communities from Northeastern Brazil: an assessment based on use value. Journal of Enviromental Management, 96:106-115.

Martins-da-Silva, RCV, Pereira, JF, Lima, HC. 2008. O gênero Copaifera (Leguminosae-Caesalpinioideae) na Amazônia Brasileira. Rodriguésia, 59: 455-476.

Melo, MC, Barbosa, RI. 2007. Árvores e arbustos das savanas de Roraima - Guia de Campo Ilustrado. $1^{a}$ ed. PMBV/ CONSEMMA, Boa Vista, 36p.

Monteiro, JM, Almeida, CFCBR, Albuquerque, UP, Lucena, RFP, Florentino, ATN, Oliveira, RLC. 2006. Use and traditional management of Anadenanthera colubrina (Vell.) Brenan in the semi-arid region of northeastern Brazil. Journal of Ethnobiology and Ethnomedicine, 2:1-7.

Oliveira, RLC, Oliveira, SKS, Scudeller, VV, Barbosa, RI. 2017a. Árvores úteis da Comunidade Darora. Boa Vista: UERR Edições. 68p.

Oliveira, RLC, Scudeller, VV, Barbosa, RI. 2017b. Uso e conhecimento tradicional de Byrsonima crassifolia e B. coccolobifolia (Malpighiaceae) em uma Comunidade Makuxi na Savana de Roraima, extremo norte do Brasil. Acta Amazonica, 47(2): 133-140.

Rigamonte Azevedo, OC. et al. 2006. Potencial de produção de óleo-resina de copaíba (Copaifera sp.) de populações naturais do sudoeste da Amazônia. Revista Árvore, 30(4): 583-91.

Veiga Junior, VF, Pinto, AC. 2002. O Gênero Copaifera L. Química nova, 25(2): 273-86. 
Vieira, RF, Silva, SR, Alves, RBN, Silva, DB, Wetzel, MMVS, Dias, TAB, Udry, MC, Martins, RC. 2002. Estratégias para Conservação e Manejo de Recursos Genéticos de Plantas Medicinais e Aromáticas: Resultados da $1^{a}$ Reunião Técnica. Brasília: Embrapa Recursos Genéticos e Biotecnologia/ Instituto Brasileiro do Meio Ambiente e dos Recursos Naturais Renováveis (IBAMA) / Conselho Nacional de Desenvolvimento Científico e Tecnológico (CNPq), 184p.

Voeks, RA. 2007. Are women reservoirs of traditional plant knowledge? Gender, ethnobotany and globalization in northeast Brazil. Singapore Journal of Tropical Geography, 28: 7-20. 